\title{
On the Undecidability of Fuzzy Description Logics with GCIs and Product t-norm
}

\author{
Franz Baader and Rafael Peñaloza \\ Theoretical Computer Science, TU Dresden, Germany \\ \{baader, penaloza\}@tcs.inf.tu-dresden.de
}

\begin{abstract}
The combination of Fuzzy Logics and Description Logics (DLs) has been investigated for at least two decades because such fuzzy DLs can be used to formalize imprecise concepts. In particular, tableau algorithms for crisp Description Logics have been extended to reason also with their fuzzy counterparts. Recently, it has been shown that, in the presence of general concept inclusion axioms (GCIs), some of these fuzzy DLs actually do not have the finite model property, thus throwing doubt on the correctness of tableau algorithm for which it was claimed that they can handle fuzzy DLs with GCIs.

In a previous paper, we have shown that these doubts are indeed justified, by proving that a certain fuzzy DL with product t-norm and involutive negation is undecidable. In the present paper, we show that undecidability also holds if we consider a t-norm-based fuzzy DL where disjunction and involutive negation are replaced by the constructor implication, which is interpreted as the residuum. The only condition on the t-norm is that it is a continuous t-norm "starting" with the product t-norm, which covers an uncountable family of t-norms.
\end{abstract}

\section{Introduction}

Description logics (DLs) [1] are a family of logic-based knowledge representation formalisms, which can be used to represent the conceptual knowledge of an application domain in a structured and formally well-understood way. They were employed in various application domains, such as natural language processing, configuration, and databases, but their main breakthrough arguably came with the adoption of the DL-based language OWL [17] as standard ontology language for the semantic web. Another successful application area for DLs is the definition of medical ontologies, such as Snomed CT ${ }^{1}$ and Galen. ${ }^{2}$

In Description Logics, concepts are formally described by concept descriptions, i.e., expressions that are built from concept names (unary predicates) and role names (binary predicates) using concept constructors. The expressivity of a particular DL is determined by which concept constructors are available in it. From a semantic point of view, concept names and concept descriptions

${ }^{1}$ http://www.ihtsdo.org/snomed-ct/

${ }^{2}$ http://www.opengalen.org/ 
represent sets of individuals, whereas roles represent binary relations between individuals. For example, using the concept names Patient and Running-nose, and the role name has-symptom, the concept of all patients with running noses can be represented by the concept description

\section{Patient $\sqcap \exists$ has-symptom.Running-nose.}

In addition to the description language (i.e., the formalism for constructing concept descriptions), DLs provide their users with a terminological and an assertional formalism. In its simplest form, a DL terminology (usually called TBox) can be used to introduce abbreviations for complex concept descriptions. For example, the concept definition

$$
\text { Private-patient } \equiv \text { Patient } \sqcap \exists \text { has-insurance.Private-health }
$$

says that private patients are patients that have a private health insurance. So-called general concept inclusions (GCIs) can be used to state additional constraints on the interpretation of concepts and roles. In our medical example, one could express that patients with running noses have a cold or hay fever using the GCI

Patient $\sqcap \exists$ has-symptom.Running-nose $\sqsubseteq \exists$ has-disease.(Cold $\sqcup$ Hay-fever).

Note that the concept definition $A \equiv C$ can be expressed using the two GCIs $A \sqsubseteq C$ and $C \sqsubseteq A$.

In the assertional part (ABox) of a DL-based ontology, facts about a specific application situation can be stated, by introducing named individuals and relating them to concepts and roles. For example, the assertions

LINDA : Patient, (LINDA, AXA-PPP) : has-insurance, AXA-PPP : Private-health

state that Linda is a patient that has the private health insurance AXA-PPP. An ontology is a TBox together with an ABox, i.e., finite set of GCIs and assertions.

Knowledge representation systems based on DLs provide their users with various inference services that allow them to deduce implicit knowledge from the explicitly represented knowledge. For example, given the concept definition and the assertions of our example, one can deduce the assertion LINDA : Private-patient, i.e., that Linda is a private patient. An important inference service for DL-based ontologies is testing their consistency, i.e., checking whether a given ontology is non-contradictory by testing whether it has a model. In fact, all the other standard inference problems can be reduced to consistency.

Fuzzy variants of Description Logics (DLs) were introduced in order to deal with applications where membership to concepts cannot always be determined in a precise way. For example, assume that we want to express that a patient that has a high temperature and a running nose has a cold using the GCI

Patient $\sqcap \exists$ has-symptom.Running-nose $\rceil \exists$ has-temperature.High $\sqsubseteq \exists$ has-disease.Cold. 
Here it makes sense to view High as a fuzzy concept, to which $36^{\circ} \mathrm{C}$ belongs with a low membership degree (say 0.2 ), $38^{\circ} \mathrm{C}$ with a higher membership degree (say 0.7), and $40^{\circ} \mathrm{C}$ with an even higher membership degree (say 0.9). In the presence of such fuzzy concepts, ABox assertions must then be equipped with a membership degree. For example, the assertion $\left\langle T_{1}\right.$ : High $\left.\geq 0.8\right\rangle$ says that temperature $T_{1}$ is high with membership degree at least 0.8 . If we are not so sure about the measurement (e.g., if it was taken under the armpit), we could also equip the role assertion (LINDA, $\mathrm{T}_{1}$ ) : has-temperature with a membership degree smaller than 1 . The use of fuzzy concepts in medical applications is, for instance, described in more detail in [19].

A great variety of fuzzy DLs have been investigated in the literature $[18,14]$. In fact, compared to crisp DLs, fuzzy DLs offer an additional degree of freedom when defining their expressiveness: in addition to deciding which concept constructors (like conjunction $\sqcap$, disjunction $\sqcup$, existential restriction $\exists r . C$ ) and which terminological formalism (like no TBox, acyclic concept definitions, general concept inclusions) to use, one must also decide how to interpret the concept constructors by appropriate functions on the domain of fuzzy values $[0,1]$. For example, conjunction can be interpreted by different t-norms (such as Gödel, Łukasiewicz, and product) and there are also different options for how to interpret negation (such as involutive negation and residual negation). In addition, one can either consider all models or only so-called witnessed models [16] when defining the semantics of fuzzy DLs.

Decidability of fuzzy DLs is often shown by adapting the tableau-based algorithms [3] for the corresponding crisp DL to the fuzzy case. This was first done for the case of DLs without general concept inclusion axioms (GCIs) $[26,24,22,9]$, but then also extended to GCIs $[23,25,7,8]$. Usually, these tableau algorithm reason w.r.t. witnessed models. ${ }^{3}$ It should be noted, however, that in the presence of GCIs there are different ways of extending the notion of witnessed models from [16], depending on whether the witnessed property is required to apply also to GCIs (in which case we talk about strongly witnessed models) or not (in which case we talk about witnessed models).

The paper [7] considers the case of reasoning w.r.t. fuzzy GCIs in the setting of a logic with product t-norm and involutive negation. More precisely, the tableau algorithm introduced in that paper is supposed to check whether an ontology consisting of fuzzy GCIs and fuzzy ABox assertions expressed in this DL has a strongly witnessed model or not. ${ }^{4}$ Actually, the proof of correctness of this algorithm given in [7] implies that, whenever such an ontology has a strongly witnessed model, then it has a finite model. However, it was recently shown in [4] that this is not the case in the presence of general concept inclusion axioms, i.e., there is an ontology written in this logic that has a strongly witnessed model, but does not have a finite model. Of course, this does not automatically imply

\footnotetext{
${ }^{3}$ In fact, witnessed models were introduced in [16] to correct the proof of correctness for the tableau algorithm presented in [26].

${ }^{4}$ Note that the authors of [7] actually use the term "witnessed models" for what we call "strongly witnessed models."
} 
that the algorithm itself is wrong. In fact, if one applies the algorithm from [7] to the ontology used in [4] to demonstrate the failure of the finite model property, then one obtains the correct answer, and in [4] the authors actually conjecture that the algorithm is still correct. However, incorrectness of the algorithm has now independently been shown in [5] and in [2]. Thus, one can ask whether the fuzzy DL considered in [7] is actually decidable. Though this question is not answered in [2], the paper gives strong indications that the answer might in fact be "no." More precisely, [2] contains a proof of undecidability for a variant of the fuzzy DL considered in [7], which (i) additionally allows for strict GCIs, i.e., GCIs whose fuzzy value is required to be strictly greater than a given rational number; and (ii) where the notion of strongly witnessed models used in [7] is replaced by the weaker notion of witnessed models.

In the present paper, we show that, in the presence of GCIs, undecidability also holds if we consider a t-norm-based fuzzy DL where disjunction and involutive negation are replaced by the constructor implication, which is interpreted as the residuum. ${ }^{5}$ The only condition on the t-norm is that it is a continuous t-norm "starting" with the product t-norm. In particular, this includes the fuzzy DL with product t-norm introduced in [16], where decidability of reasoning w.r.t. witnessed models was shown for the case without GCIs. In [13], an analogous decidability result was shown for the case of reasoning w.r.t. so-called quasi-witnessed models. Following [13], we call this logic $*-\mathcal{A L E}$. Note that our undecidability result holds for several variants of the notion of witnessed models (including witnessed, quasi-witnessed, and strongly witnessed models).

In the next section, we introduce basic notions from fuzzy logics, and in Section 3 we introduce the fuzzy DLs considered in this paper. In Section 4 we then show undecidability of these DLs w.r.t. witnessed and quasi-witnessed models, and in Section 5 w.r.t. strongly witnessed and finite models.

\section{T-norms and Fuzzy Logic}

Fuzzy logics are formalisms introduced to express imprecise or vague information [15]. They extend classical logic by interpreting predicates as fuzzy sets over an interpretation domain. Given a non-empty domain $\Delta$, a fuzzy set is a function $F: \Delta \rightarrow[0,1]$ from $\Delta$ into the real unit interval $[0,1]$, with the intuition that an element $\delta \in \Delta$ belongs to $F$ with degree $F(\delta)$. The interpretation of the logical constructors is based on appropriate truth functions that generalize the properties of the connectives of classical logic to the interval $[0,1]$. The most prominent truth functions used in the fuzzy logic literature are based on t-norms.

A $t$-norm is a binary operator $\otimes:[0,1] \times[0,1] \rightarrow[0,1]$ that is associative and commutative, has 1 as its unit element, and is monotonic, i.e., for every $x, y, z \in$ $[0,1]$, if $x \leq y$, then $x \otimes z \leq y \otimes z$. The t-norm $\otimes$ is continuous if it is continuous

\footnotetext{
5 This change of the constructors used is not irrelevant: in general, disjunction and involutive negation cannot be expressed using only conjunction and residua.
} 


\begin{tabular}{lll} 
Name & t-norm $(x \otimes y)$ & Residuum $(x \Rightarrow y)$ \\
\hline Gödel & $\min (x, y)$ & $\begin{cases}1 & \text { if } x \leq y \\
y & \text { otherwise }\end{cases}$ \\
product & $x \cdot y$ & $\begin{cases}1 & \text { if } x \leq y \\
y / x & \text { otherwise }\end{cases}$ \\
Eukasiewicz & $\max (x+y-1,0)$ & $\min (1-x+y, 1)$
\end{tabular}

Table 1. Gödel, product and Łukasiewicz t-norms and their residua

as a function, i.e., we have for all convergent sequences $\left\{x_{n}\right\}_{n \geq 0},\left\{y_{n}\right\}_{n \geq 0}$ that

$$
\left(\lim _{n \rightarrow \infty} x_{n}\right) \otimes\left(\lim _{n \rightarrow \infty} y_{n}\right)=\lim _{n \rightarrow \infty}\left(x_{n} \otimes y_{n}\right) .
$$

If $\otimes$ is a continuous t-norm, then there exists a unique binary operator $\Rightarrow$, called the residuum, that satisfies $z \leq x \Rightarrow y$ iff $x \otimes z \leq y$ for every $x, y, z \in[0,1]$. Three important continuous t-norms are the Gödel, product and Łukasiewicz t-norms. These t-norms and their corresponding residua are shown in Table 1.

The following are simple consequences of the definition of t-norms and their residua (see [15], Lemma 2.1.6).

Lemma 1. For every $t$-norm $\otimes$ and $x, y \in[0,1]$ the following hold:

$-x \Rightarrow y=1$ iff $x \leq y$,

$-1 \Rightarrow y=y, 0 \Rightarrow y=1$, and

- if $x>0$, then $x \Rightarrow 0=0$.

The t-norms described in Table 1 are fundamental in the sense that all other continuous t-norms can be constructed from them: every continuous t-norm can be expressed as the ordered sum of copies of Łukasiewicz, Gödel and product t-norms [20]. More formally, if $\otimes$ is a continuous t-norm, then there exists a (possibly infinite) family $\mathcal{S}=\left\{\left\langle\left(a_{i}, b_{i}\right), \otimes_{i}\right\rangle \mid i \in \mathcal{J}\right\}$, where $\left(a_{i}, b_{i}\right)$ are non-empty, pairwise disjoint open subintervals of $[0,1]$ and $\otimes_{i}$ is either the Eukasiewicz or the product t-norm, such that

$$
x \otimes y= \begin{cases}a_{i}+\left(b_{i}-a_{i}\right) \cdot\left(\frac{x-a_{i}}{b_{i}-a_{i}} \otimes_{i} \frac{y-a_{i}}{b_{i}-a_{i}}\right) & \text { if } x, y \in\left[a_{i}, b_{i}\right] \text { for some } i \in \mathcal{J} \\ \min (x, y) & \text { otherwise }\end{cases}
$$

holds for all $x, y \in[0,1]$. The residuum of this t-norm is given, for every $x, y \in$ $[0,1]$, by

$$
x \Rightarrow y= \begin{cases}1 & \text { if } x \leq y \\ a_{i}+\left(b_{i}-a_{i}\right) \cdot\left(\frac{x-a_{i}}{b_{i}-a_{i}} \Rightarrow_{i} \frac{y-a_{i}}{b_{i}-a_{i}}\right) & \text { if } a_{i} \leq y<x \leq b_{i} \text { for some } i \in \mathcal{J} \\ y & \text { otherwise }\end{cases}
$$

where $\Rightarrow_{i}$ represents the residuum of the t-norm $\otimes_{i}, i \in \mathcal{J}$.

In this paper we will focus on t-norms whose expression as an ordered sum use the product t-norm as "first element." 
Definition 2. Given a $t$-norm $\otimes$ obtained as ordered sum from the family $\mathcal{S}=$ $\left\{\left\langle\left(a_{i}, b_{i}\right), \otimes_{i}\right\rangle \mid i \in \mathcal{J}\right\}$ and a number $q \in(0,1]$, we say that $\otimes q$-starts with the product t-norm (q-starts with $\Pi$ for short) if there is an index $i \in \mathcal{J}$ such that $\left(a_{i}, b_{i}\right)=(0, q)$ and $\otimes_{i}$ is the product $t$-norm. It starts with $\Pi$ if it $q$-starts with $\Pi$ for some $q \in(0,1]$.

Notice that, for every $q \in(0,1)$, there exist uncountably many t-norms that $q$-start with $\Pi$. In fact, for every real number $r \in(q, 1]$, we can take the family $\left\{\left\langle(0, q), \otimes_{1}\right\rangle,\left\langle(q, r), \otimes_{2}\right\rangle\right\}$ where $\otimes_{1}$ is the product t-norm and $\otimes_{2}$ is the Łukasiewicz t-norm. As a simple consequence of this, there are uncountably many continuous t-norms that $q$-start with $\Pi$ for a rational number $q$. Our undecidability proofs will only deal with such t-norms. The following lemma is a simple consequence of the properties described before.

Lemma 3. For a given $t$-norm $\otimes$ and $q \in(0,1]$, if $\otimes q$-starts with $\Pi$, then for every $x, y \in[0, q]$ the following holds:

$-x \otimes y=(x \cdot y) / q$, and

- if $x>y$, then $x \Rightarrow y=q \cdot(y / x)$.

\section{$3 \quad$ Fuzzy Description Logics}

In this section, we introduce the fuzzy description logic $*-\mathcal{A} \mathcal{L} \mathcal{E}$ and some of its properties, which will be useful throughout the paper.

The syntax of this logic is slightly different from standard description logics, as it has an implication constructor, but no negation or disjunction constructors. *-ALE concepts are built through the syntactic rule

$$
C::=A|\perp| \top\left|C_{1} \sqcap C_{2}\right| C_{1} \rightarrow C_{2}|\exists r . C| \forall r . C
$$

where $A$ is a concept name and $r$ is a role name.

$\mathrm{A} *-\mathcal{A L E} A B o x$ is a finite set of assertion axioms of the form $\langle a: C \triangleright q\rangle$ or $\langle(a, b): r \triangleright q\rangle$, where $C$ is a $*-\mathcal{A L E}$ concept, $r \in \mathcal{N}_{\mathcal{R}}, q$ is a rational number in the interval $[0,1], a, b$ are individual names and $\triangleright$ is either $\geq$ or $=. \mathrm{A} *-\mathcal{A L E}$ $T B o x$ is a finite set of concept inclusion axioms of the form $\langle C \sqsubseteq D \geq q\rangle$, where $C, D$ are $*-\mathcal{A L E}$ concepts and $q$ is a rational number in $[0,1]$. A $*-\mathcal{A} \mathcal{L} \mathcal{E}$ ontology is a tuple $(\mathcal{A}, \mathcal{T})$, where $\mathcal{A}$ is a $*-\mathcal{A L E}$ ABox and $\mathcal{T}$ a $*-\mathcal{A} \mathcal{L} \mathcal{E}$ TBox. For the rest of the paper we will often drop the prefix $*-\mathcal{A L E}$, and speak simply of e.g. TBoxes and ontologies.

The semantics of this logic extend the classical DL semantics by interpreting concepts and roles as fuzzy sets over an interpretation domain. The precise semantics depends on the t-norm chosen; thus, in the following, we assume that we have an arbitrary, but fixed, continuous t-norm $\otimes$ and that $\Rightarrow$ is the associated residuum. The semantics of $*-\mathcal{A L E}$ is based on interpretations. An interpretation is a tuple $\mathcal{I}=\left(\Delta^{\mathcal{I}},{ }^{\mathcal{I}}\right)$ where $\Delta^{\mathcal{I}}$ is a non-empty set, called the domain, and the function ${ }^{\mathcal{I}}$ maps each individual name $a$ to an element of $\Delta^{\mathcal{I}}$, each 
concept name $A$ to a function $A^{\mathcal{I}}: \Delta^{\mathcal{I}} \rightarrow[0,1]$ and each role name $r$ to a function $r^{\mathcal{I}}: \Delta^{\mathcal{I}} \times \Delta^{\mathcal{I}} \rightarrow[0,1]$. The interpretation function is extended to arbitrary *- $\mathcal{A} \mathcal{L} \mathcal{E}$ concepts as follows. For every $\delta \in \Delta^{\mathcal{I}}$,

$$
\begin{aligned}
\top^{\mathcal{I}}(\delta) & =1, \\
\perp^{\mathcal{I}}(\delta) & =0, \\
\left(C_{1} \sqcap C_{2}\right)^{\mathcal{I}}(\delta) & =C_{1}^{\mathcal{I}}(\delta) \otimes C_{2}^{\mathcal{I}}(\delta) \\
\left(C_{1} \rightarrow C_{2}\right)^{\mathcal{I}}(\delta) & =C_{1}^{\mathcal{I}}(\delta) \Rightarrow C_{2}^{\mathcal{I}}(\delta) \\
(\exists r . C)^{\mathcal{I}}(\delta) & =\sup _{\gamma \in \Delta^{\mathcal{I}}} r^{\mathcal{I}}(\delta, \gamma) \otimes C^{\mathcal{I}}(\gamma) \\
(\forall r . C)^{\mathcal{I}}(\delta) & =\inf _{\gamma \in \Delta^{\mathcal{I}}} r^{\mathcal{I}}(\delta, \gamma) \Rightarrow C^{\mathcal{I}}(\gamma) .
\end{aligned}
$$

The interpretation $\mathcal{I}=\left(\Delta^{\mathcal{I}},{ }^{\mathcal{I}}\right)$ satisfies the assertional axiom $\langle a: C \triangleright q\rangle$ iff $C^{\mathcal{I}}\left(a^{\mathcal{I}}\right) \triangleright q$, it satisfies $\langle(a, b): r \triangleright q\rangle$ iff $r^{\mathcal{I}}\left(a^{\mathcal{I}}, b^{\mathcal{I}}\right) \triangleright q$ and it satisfies the concept

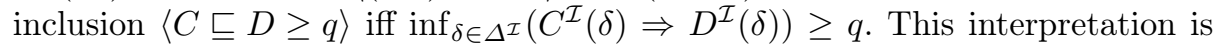
called a model of the ontology $\mathcal{O}$ if it satisfies all the axioms in $\mathcal{O}$.

In fuzzy DLs, reasoning is often restricted to witnessed models [16]. An interpretation $\mathcal{I}$ is called witnessed if it satisfies the following two conditions:

(wit1) for every $\delta \in \Delta^{\mathcal{I}}$, role $r$ and concept $C$ there exists $\gamma \in \Delta^{\mathcal{I}}$ such that $(\exists r . C)^{\mathcal{I}}(\delta)=r^{\mathcal{I}}(\delta, \gamma) \cdot C^{\mathcal{I}}(\gamma)$, and

(wit2) for every $\delta \in \Delta^{\mathcal{I}}$, role $r$ and concept $C$ there exists $\gamma \in \Delta^{\mathcal{I}}$ such that $(\forall r . C)^{\mathcal{I}}(\delta)=r^{\mathcal{I}}(\delta, \gamma) \Rightarrow C^{\mathcal{I}}(\gamma)$.

This model is called weakly witnessed if it satisfies (wit1) and quasi-witnessed if it satisfies (wit1) and the condition

(wit2') for every $\delta \in \Delta^{\mathcal{I}}$, role $r$ and concept $C$, either $(\forall r . C)^{\mathcal{I}}=0$ or there exists $\gamma \in \Delta^{\mathcal{I}}$ such that $(\forall r . C)^{\mathcal{I}}(\delta)=r^{\mathcal{I}}(\delta, \gamma) \Rightarrow C^{\mathcal{I}}(\gamma)$.

In the presence of GCIs, witnessed interpretations are sometimes further restricted $[9,4,14]$ to satisfy

(wit3) for every two concepts $C, D$, there is a $\gamma$ such that

$$
\inf _{\eta \in \Delta^{\mathcal{I}}}\left(C^{\mathcal{I}}(\eta) \Rightarrow D^{\mathcal{I}}(\eta)\right)=C^{\mathcal{I}}(\gamma) \Rightarrow D^{\mathcal{I}}(\gamma) .
$$

Witnessed interpretations that satisfy this third restriction (wit3) are called strongly witnessed interpretations.

We say that an ontology $\mathcal{O}$ is consistent (resp. weakly witnessed consistent, quasi-witnessed consistent, witnessed consistent, strongly witnessed consistent) if it has a model (resp. a weakly witnessed model, a quasi-witnessed model, a witnessed model, a strongly witnessed model). Obviously, strongly witnessed consistency implies witnessed consistency, which implies quasi-witnessed consistency, which itself implies weakly witnessed consistency. The converse implications, however, need not hold; for instance, a quasi-witnessed consistent $*-\mathcal{A L E}$ ontology that has no witnessed models can be derived from the example in [13]. 
Witnessed models were introduced to simplify the construction of tableaubased reasoning procedures for fuzzy DLs [16]. Intuitively, with the general semantics for existential restrictions, interpreted as a supremum, it is possible that an existential restriction is interpreted with a value that is never reached; that is, $(\exists r . C)^{\mathcal{I}}(\delta)>r^{\mathcal{I}}(\delta, \eta) \otimes C^{\mathcal{I}}(\eta)$ for all $\eta \in \Delta^{\mathcal{I}}$. Given an existential restriction, a tableau-based procedure tries to introduce one successor individual that yields this value. Condition (wit1) ensures that this approach is sound. Without it, the procedure would also need to address the case where there are infinitely many successors yielding values whose supremum is the value of the existential restriction. It is not clear how to do this with a terminating procedure.

We now derive some properties of the $*-\mathcal{A L E}$ axioms and introduce useful abbreviations. First, recall that, for all $x, y \in[0,1]$, it holds that $x \Rightarrow y=1$ iff $x \leq y$ (Lemma 1). Thus, given two concepts $C, D$, the axiom $\langle C \sqsubseteq D \geq 1\rangle$ expresses that $C^{\mathcal{I}}(\delta) \leq D^{\mathcal{I}}(\delta)$ for all $\delta \in \Delta^{\mathcal{I}}$.

In the following, we will use the expression $\langle C \stackrel{r}{\rightsquigarrow} D\rangle$ to abbreviate the axioms $\langle C \sqsubseteq \forall r . D \geq 1\rangle,\langle\exists r . D \sqsubseteq C \geq 1\rangle$. To understand this abbreviation, consider an interpretation $\mathcal{I}$ satisfying $\langle C \stackrel{r}{\sim} D\rangle$ and let $\delta, \gamma \in \Delta^{\mathcal{I}}$ with $r^{\mathcal{I}}(\delta, \gamma)=1$. From the first axiom it follows that

$$
\begin{aligned}
C^{\mathcal{I}}(\delta) \leq(\forall r . D)^{\mathcal{I}}(\delta) & =\inf _{\eta \in \Delta^{\mathcal{I}}} r^{\mathcal{I}}(\delta, \eta) \Rightarrow D^{\mathcal{I}}(\eta) \\
& \leq r^{\mathcal{I}}(\delta, \gamma) \Rightarrow D^{\mathcal{I}}(\gamma)=1 \Rightarrow D^{\mathcal{I}}(\gamma)=D^{\mathcal{I}}(\gamma) .
\end{aligned}
$$

From the second axiom it follows that

$$
\begin{aligned}
C^{\mathcal{I}}(\delta) \geq(\exists r . D)^{\mathcal{I}}(\delta) & =\sup _{\eta \in \Delta^{\mathcal{I}}} r^{\mathcal{I}}(\delta, \eta) \otimes D^{\mathcal{I}}(\eta) \\
& \geq r^{\mathcal{I}}(\delta, \gamma) \otimes D^{\mathcal{I}}(\gamma)=1 \otimes D^{\mathcal{I}}(\gamma)=D^{\mathcal{I}}(\gamma),
\end{aligned}
$$

and hence, both axioms together imply that $C^{\mathcal{I}}(\delta)=D^{\mathcal{I}}(\gamma)$. In other words, $\langle C \stackrel{r}{\rightsquigarrow} D\rangle$ expresses that the value of $C^{\mathcal{I}}(\delta)$ is propagated to the valuation of the concept $D$ on all $r$ successors with degree 1 of $\delta$. Conversely, given an interpretation $\mathcal{I}$ such that $r^{\mathcal{I}}(\delta, \gamma) \in\{0,1\}$ for all $\delta, \gamma \in \Delta^{\mathcal{I}}$, if $r^{\mathcal{I}}(\delta, \gamma)=1$ implies $C^{\mathcal{I}}(\delta)=D^{\mathcal{I}}(\gamma)$, then $\mathcal{I}$ is a model of $\langle C \stackrel{r}{\sim} D\rangle$.

For a concept $C$ and a natural number $n \geq 1$, the expression $C^{n}$ denotes the concatenation of $C$ with itself $n$ times, i.e., $C^{1}:=C$ and $C^{n+1}:=C \sqcap C^{n}$. If $\otimes$ $q$-starts with $\Pi$, then the semantics of $\sqcap$ yields $\left(C^{n}\right)^{\mathcal{I}}(\delta)=\left(C^{\mathcal{I}}(\delta)\right)^{n} / q^{n-1}$, for every interpretation $\mathcal{I}$ and every $\delta \in \Delta^{\mathcal{I}}$ with $C^{\mathcal{I}}(\delta) \in[0, q]$ (see Lemma 3 ).

For the rest of the paper we assume that $\otimes$ q-starts with $\Pi$ for some arbitrary but fixed rational number $q \in[0,1]$. We will show that, under such a t-norm, consistency of $*-\mathcal{A L E}$ ontologies w.r.t. the different variants of witnessed models introduced above is undecidable.

\section{Undecidability w.r.t. Witnessed Models}

We will show undecidability using a reduction from the Post correspondence problem, which is well-known to be undecidable [21]. 
Definition 4 (PCP). Let $\left(\left(v_{1}, w_{1}\right), \ldots,\left(v_{m}, w_{m}\right)\right)$ be a finite list of pairs of words over an alphabet $\Sigma=\{1, \ldots, s\}, s>1$. The Post correspondence problem (PCP) asks whether there is a non-empty sequence $i_{1}, i_{2}, \ldots, i_{k}, 1 \leq i_{j} \leq m$, such that $v_{i_{1}} v_{i_{2}} \cdots v_{i_{k}}=w_{i_{1}} w_{i_{2}} \cdots w_{i_{k}}$. If such a sequence exists, then the word $i_{1} i_{2} \cdots i_{k}$ is called $a$ solution of the problem.

We assume w.l.o.g. that there is no pair $v_{i}, w_{i}$ where both words are empty. For a word $\mu=i_{1} i_{2} \cdots i_{k} \in\{1, \ldots, m\}^{*}$, we will denote as $v_{\mu}$ and $w_{\mu}$ the words $v_{i_{1}} v_{i_{2}} \cdots v_{i_{k}}$ and $w_{i_{1}} w_{i_{2}} \cdots w_{i_{k}}$, respectively.

The alphabet $\Sigma$ consists of the first $s$ positive integers. We can thus view every word in $\Sigma^{*}$ as a natural number represented in base $s+1$ in which 0 never occurs. Using this intuition, we will express the empty word as the number 0 .

In the following reductions, we will encode the word $w$ in $\Sigma^{*}$ using the number $q \cdot 2^{-w} \in[0, q]$. We will construct an ontology whose models encode the search for a solution. The interpretation of two designated concept names $A$ and $B$ at a node will respectively correspond to the words $v_{\mu}$ and $w_{\mu}$ for $\mu \in\{1, \ldots, m\}^{*}$.

It should be noted that, in the following constructions, the only relevant values used for interpreting the different concepts will be $[0, q] \cup\{1\}$. For this reason, it is only important that $\otimes q$-starts with $\Pi$, while the precise definition of the t-norm over the rest of the unit interval is irrelevant.

To be more precise, we will show undecidability of consistency w.r.t. witnessed models by constructing, for a given instance $\mathcal{P}=\left(\left(v_{1}, w_{1}\right), \ldots,\left(v_{m}, w_{m}\right)\right)$ of the PCP, an ontology $\mathcal{O}_{\mathcal{P}}$ such that, for every witnessed model $\mathcal{I}$ of $\mathcal{O}_{\mathcal{P}}$ and every $\mu \in\{1, \ldots, m\}^{*}$, there is an element $\delta_{\mu} \in \Delta^{\mathcal{I}}$ with $A^{\mathcal{I}}\left(\delta_{\mu}\right)=q \cdot 2^{-v_{\mu}}$ and $B^{\mathcal{I}}\left(\delta_{\mu}\right)=q \cdot 2^{-w_{\mu}}$. Additionally, we will show that this ontology has a witnessed model whose domain has only these elements. Then, $\mathcal{P}$ has a solution iff for every witnessed model $\mathcal{I}$ of $\mathcal{O}_{\mathcal{P}}$ there exist a $\delta \in \Delta^{\mathcal{I}}$ such that $A^{\mathcal{I}}(\delta)=B^{\mathcal{I}}(\delta)$.

Let $\delta \in \Delta^{\mathcal{I}}$ encode the words $v, w \in \Sigma^{*}$; that is, $A^{\mathcal{I}}(\delta)=q \cdot 2^{-v}$ and $B^{\mathcal{I}}(\delta)=q \cdot 2^{-w}$, and let $i, 1 \leq i \leq m$. Assume additionally that we have concept names $V_{i}, W_{i}$ with $V_{i}^{\mathcal{I}}(\delta)=q \cdot 2^{-v_{i}}$ and $W_{i}^{\mathcal{I}}(\delta)=q \cdot 2^{-w_{i}}$. We want to ensure the existence of a node $\gamma$ that encodes the concatenation of the words $v, w$ with the $i$-th pair from $\mathcal{P}$; i.e. $v v_{i}$ and $w w_{i}$. This is done through the TBox

$$
\mathcal{T}_{\mathcal{P}}^{i}:=\left\{\left\langle\top \sqsubseteq \exists r_{i} \cdot \top \geq 1\right\rangle,\left\langle\left(V_{i} \sqcap A^{(s+1)^{\left|v_{i}\right|}}\right) \stackrel{r_{i}}{\rightsquigarrow} A\right\rangle,\left\langle\left(W_{i} \sqcap B^{(s+1)^{\left|w_{i}\right|}}\right) \stackrel{r_{i}}{\rightsquigarrow} B\right\rangle\right\} .
$$

Recall that we are viewing words in $\Sigma^{*}$ as natural numbers in base $s+1$. Thus, the concatenation of two words $u, u^{\prime}$ corresponds to the operation $u \cdot(s+1)^{\left|u^{\prime}\right|}+u^{\prime}$. Additionally, $A^{\mathcal{I}}(\delta) \leq q$ and hence

$$
\left(A^{(s+1)^{\left|v_{i}\right|}}\right)^{\mathcal{I}}(\delta)=\frac{q^{(s+1)^{\left|v_{i}\right|}} \cdot 2^{-v \cdot(s+1)^{\left|v_{i}\right|}}}{q^{(s+1)^{\left|v_{i}\right|}-1}}=q \cdot 2^{-v \cdot(s+1)^{\left|v_{i}\right|}} .
$$

Since $V_{i}^{\mathcal{I}}(\delta) \leq q$, we then have

$$
\left(V_{i} \sqcap A^{(s+1)^{\left|v_{i}\right|}}\right)^{\mathcal{I}}(\delta)=\frac{\left(q \cdot 2^{-v_{i}}\right) \cdot\left(q \cdot 2^{-v \cdot(s+1)^{\left|v_{i}\right|}}\right)}{q}=q \cdot 2^{-v v_{i}} .
$$


Analogously, we get that $\left(W_{i} \sqcap B^{(s+1)^{\left|w_{i}\right|}}\right)^{\mathcal{I}}(\delta)=q \cdot 2^{-w w_{i}}$.

If $\mathcal{I}$ is a witnessed model of $\mathcal{T}_{\mathcal{P}}^{i}$, then from the first axiom in $\mathcal{T}_{\mathcal{P}}^{i}$ it follows that $\left(\exists r_{i} \cdot \top\right)^{\mathcal{I}}(\delta)=1$, and according to (wit1), there must exist a $\gamma \in \Delta^{\mathcal{I}}$ with $r^{\mathcal{I}}(\delta, \gamma)=1$. The last two axioms then ensure that $A^{\mathcal{I}}(\gamma)=q \cdot 2^{-v v_{i}}$ and $B^{\mathcal{I}}(\gamma)=q \cdot 2^{-w w_{i}}$; thus, the concept names $A$ and $B$ encode, at node $\gamma$, the words $v v_{i}$ and $w w_{i}$, as desired. If we want to use this construction to recursively construct all the pairs of concatenated words defined by $\mathcal{P}$, we need to ensure also that $V_{j}^{\mathcal{I}}(\gamma)=q \cdot 2^{-v_{j}}, W_{j}^{\mathcal{I}}(\gamma)=q \cdot 2^{-w_{j}}$ hold for every $j, 1 \leq j \leq m$. This can be done through the axioms

$$
\mathcal{T}_{\mathcal{P}}^{0}:=\left\{\left\langle V_{j} \stackrel{r_{i}}{\rightsquigarrow} V_{j}\right\rangle,\left\langle W_{j} \stackrel{r_{i}}{\rightsquigarrow} W_{j}\right\rangle \mid 1 \leq i, j \leq m\right\} .
$$

It only remains to ensure that there is a node $\delta_{\varepsilon}$ where

$$
A^{\mathcal{I}}\left(\delta_{\varepsilon}\right)=B^{\mathcal{I}}\left(\delta_{\varepsilon}\right)=q=q \cdot 2^{0},
$$

that is, where $A$ and $B$ encode the empty word, and for every $j, 1 \leq i \leq m$, $V_{j}^{\mathcal{I}}\left(\delta_{\varepsilon}\right)=q \cdot 2^{-v_{j}}$ and $W_{j}^{\mathcal{I}}\left(\delta_{\varepsilon}\right)=q \cdot 2^{-w_{j}}$ hold. This condition is easily enforced through the ABox

$$
\begin{aligned}
\mathcal{A}_{\mathcal{P}}^{0}:= & \{\langle a: A=q\rangle,\langle a: B=q\rangle\} \cup \\
& \left\{\left\langle a: V_{i}=q \cdot 2^{-v_{i}}\right\rangle,\left\langle a: W_{i}=q \cdot 2^{-w_{i}}\right\rangle \mid 1 \leq i \leq m\right\} .
\end{aligned}
$$

Finally, we include a concept name $H$ that must be interpreted as $q / 2$ in every domain element reachable from $a$. This is enforced by the following axioms:

$$
\begin{aligned}
\mathcal{A}_{0} & :=\{\langle a: H=q / 2\rangle\}, \\
\mathcal{T}_{0} & :=\left\{\left\langle H \stackrel{r_{i}}{\rightsquigarrow} H\right\rangle \mid 1 \leq i \leq m\right\} .
\end{aligned}
$$

The concept name $H$ will later be used to detect whether $\mathcal{P}$ has a solution (see the proof of Theorem 6).

Let now $\mathcal{O}_{\mathcal{P}}:=\left(\mathcal{A}_{\mathcal{P}}, \mathcal{T}_{\mathcal{P}}\right)$ where $\mathcal{A}_{\mathcal{P}}=\mathcal{A}_{\mathcal{P}}^{0} \cup \mathcal{A}_{0}$ and $\mathcal{T}_{\mathcal{P}}:=\mathcal{T}_{0} \cup \bigcup_{i=0}^{m} \mathcal{T}_{\mathcal{P}}^{i}$. We define the interpretation $\mathcal{I}_{\mathcal{P}}:=\left(\Delta^{\mathcal{I}_{\mathcal{P}}}, \mathcal{I}_{\mathcal{P}}\right)$ as follows:

$$
\begin{aligned}
& -\Delta^{\mathcal{I}_{\mathcal{P}}}=\{1, \ldots, m\}^{*}, \\
& -a^{\mathcal{I}_{\mathcal{P}}}=\varepsilon,
\end{aligned}
$$

for every $\mu \in \Delta^{\mathcal{I}_{\mathcal{P}}}$,

$$
-A^{\mathcal{I}_{\mathcal{P}}}(\mu)=q \cdot 2^{-v_{\mu}}, B^{\mathcal{I}_{\mathcal{P}}}(\mu)=q \cdot 2^{-w_{\mu}}, H^{\mathcal{I}_{\mathcal{P}}}(\mu)=q / 2,
$$

and for all $j, 1 \leq j \leq m$,

$$
\begin{aligned}
& -V_{j}^{\mathcal{I}_{\mathcal{P}}}(\mu)=q \cdot 2^{-v_{j}}, W_{j}^{\mathcal{I}_{\mathcal{P}}}(\mu)=q \cdot 2^{-w_{j}}, \text { and } \\
& -r_{j}^{\mathcal{I}_{\mathcal{P}}}(\mu, \mu j)=1 \text { and } r_{j}^{\mathcal{I}_{\mathcal{P}}}\left(\mu, \mu^{\prime}\right)=0 \text { if } \mu^{\prime} \neq \mu j .
\end{aligned}
$$

It is easy to see that $\mathcal{I}_{\mathcal{P}}$ is in fact a model of $\mathcal{O}_{\mathcal{P}}$. This model is trivially witnessed since, for every $i, 1 \leq i \leq m$, every node has only one $r_{i}$ successor with degree greater than 0. More interesting, however, is that every witnessed model $\mathcal{I}$ of $\mathcal{O}_{\mathcal{P}}$ "contains" $\mathcal{I}_{\mathcal{P}}$ in the sense stated in the following lemma. 
Lemma 5. Let $\mathcal{I}$ be a witnessed model of $\mathcal{O}_{\mathcal{P}}$. Then there exists a function $f: \Delta^{\mathcal{I}_{\mathcal{P}}} \rightarrow \Delta^{\mathcal{I}}$ such that, for every $\mu \in \Delta^{\mathcal{I}_{\mathcal{P}}}, C^{\mathcal{I}_{\mathcal{P}}}(\mu)=C^{\mathcal{I}}(f(\mu))$ holds for every concept name $C$ and $r_{i}^{\mathcal{I}}(f(\mu), f(\mu i))=1$ holds for every $i, 1 \leq i \leq m$.

Proof. The function $f$ is built inductively on the length of $\mu$. First, as $\mathcal{I}$ is a model of $\mathcal{A}_{\mathcal{P}}$, there must be a $\delta \in \Delta^{\mathcal{I}}$ such that $a^{\mathcal{I}}=\delta$. Notice that $\mathcal{A}_{\mathcal{P}}$ fixes the interpretation of all concept names on $\delta$ and hence $f(\varepsilon)=\delta$ satisfies the condition of the lemma.

Let now $\mu$ be such that $f(\mu)$ has already been defined. By induction, we can assume that $A^{\mathcal{I}}(f(\mu))=q \cdot 2^{-v_{\mu}}, B^{\mathcal{I}}(f(\mu))=q \cdot 2^{-w_{\mu}}, H^{\mathcal{I}}(f(\mu))=q / 2$, and for every $j, 1 \leq j \leq m, V_{j}^{\mathcal{I}}(f(\mu))=q \cdot 2^{-v_{j}}, W_{j}^{\mathcal{I}}(f(\mu))=q \cdot 2^{-w_{j}}$. Since $\mathcal{I}$ is a witnessed model of $\left\langle\top \sqsubseteq \exists r_{i} \cdot \top \geq 1\right\rangle$, for all $i, 1 \leq i \leq m$, there exists a $\gamma \in \Delta^{\mathcal{I}}$ with $r_{i}^{\mathcal{I}}(f(\mu), \gamma)=1$, and as $\mathcal{I}$ satisfies all the axioms of the form $\langle C \stackrel{r}{\rightsquigarrow} D\rangle$ in $\mathcal{T}_{\mathcal{P}}$, it follows that

$$
A^{\mathcal{I}}(\gamma)=q \cdot 2^{-v_{\mu} v_{i}}=q \cdot 2^{-v_{\mu i}}, \quad B^{\mathcal{I}}(\gamma)=q \cdot 2^{-w_{\mu} w_{i}}=q \cdot 2^{-w_{\mu i}}, \quad H^{\mathcal{I}}(\gamma)=q / 2,
$$

and for all $j, 1 \leq j \leq m, V_{j}^{\mathcal{I}}(\gamma)=q \cdot 2^{-v_{j}}, W_{j}^{\mathcal{I}}(\gamma)=q \cdot 2^{-w_{j}}$. Setting $f(\mu i)=\gamma$ thus satisfies the required property.

From this lemma it then follows that, if the $\mathrm{PCP} \mathcal{P}$ has a solution $\mu$ for some $\mu \in\{1, \ldots, m\}^{+}$, then every witnessed model $\mathcal{I}$ of $\mathcal{O}_{\mathcal{P}}$ contains a node $\delta=f(\mu)$ such that $A^{\mathcal{I}}(\delta)=B^{\mathcal{I}}(\delta)$; i.e., where $A$ and $B$ encode the same word. Conversely, if every witnessed model contains such a node, then in particular $\mathcal{I}_{\mathcal{P}}$ does, and thus $\mathcal{P}$ has a solution. The question is now how to detect whether a node with this characteristics exists in every model. We will extend $\mathcal{O}_{\mathcal{P}}$ with axioms that further restrict $\mathcal{I}_{\mathcal{P}}$ to satisfy $A^{\mathcal{I}_{\mathcal{P}}}(\mu) \neq B^{\mathcal{I}_{\mathcal{P}}}(\mu)$ for every $\mu \in\{1, \ldots, m\}^{+}$. This ensures that the extended ontology has a model iff $\mathcal{P}$ has no solution.

In order to come up with the right axioms for achieving this, suppose for now that, for some $\mu \in\{1, \ldots, m\}^{*}$, it holds that

$$
q \cdot 2^{-v_{\mu}}=A^{\mathcal{I}_{\mathcal{P}}}(\mu)>B^{\mathcal{I}_{\mathcal{P}}}(\mu)=q \cdot 2^{-w_{\mu}} .
$$

We then have that $v_{\mu}<w_{\mu}$ and hence $w_{\mu}-v_{\mu} \geq 1$. It thus follows that

$$
(A \rightarrow B)^{\mathcal{I}_{\mathcal{P}}}(\mu)=q \cdot\left(q \cdot 2^{-w_{\mu}}\right) /\left(q \cdot 2^{-v_{\mu}}\right)=q \cdot 2^{-\left(w_{\mu}-v_{\mu}\right)} \leq q \cdot 2^{-1}=q / 2
$$

and thus $((A \rightarrow B) \sqcap(B \rightarrow A))^{\mathcal{I}_{\mathcal{P}}}(\mu) \leq q / 2$. Likewise, if $A^{\mathcal{I}_{\mathcal{P}}}(\mu)<B^{\mathcal{I}_{\mathcal{P}}}(\mu)$, we also get $((A \rightarrow B) \sqcap(B \rightarrow A))^{\mathcal{I}_{\mathcal{P}}}(\mu) \leq q / 2$. Additionally, if $A^{\mathcal{I}_{\mathcal{P}}}(\mu)=B^{\mathcal{I}_{\mathcal{P}}}(\mu)$, then it is easy to verify (see Lemma 1$)$ that $((A \rightarrow B) \sqcap(B \rightarrow A))^{\mathcal{I}_{\mathcal{P}}}(\mu)=1$. From all this it follows that, for every $\mu \in\{1, \ldots, m\}^{*}$,

$$
A^{\mathcal{I}_{\mathcal{P}}}(\mu) \neq B^{\mathcal{I}_{\mathcal{P}}}(\mu) \quad \text { iff } \quad((A \rightarrow B) \sqcap(B \rightarrow A))^{\mathcal{I}_{\mathcal{P}}}(\mu) \leq q / 2 .
$$

Thus, the instance $\mathcal{P}$ has no solution iff for every $\mu \in\{1, \ldots, m\}^{+}$it holds that $((A \rightarrow B) \sqcap(B \rightarrow A))^{\mathcal{I}_{\mathcal{P}}}(\mu) \leq q / 2$.

We define now the ontology $\mathcal{O}_{\mathcal{P}}^{\prime}:=\left(\mathcal{A}_{\mathcal{P}}, \mathcal{T}_{\mathcal{P}}^{\prime}\right)$ where

$$
\mathcal{T}_{\mathcal{P}}^{\prime}:=\mathcal{T}_{\mathcal{P}} \cup\left\{\left\langle\top \sqsubseteq \forall r_{i} .(((A \rightarrow B) \sqcap(B \rightarrow A)) \rightarrow H) \geq 1\right\rangle \mid 1 \leq i \leq m\right\} .
$$


Theorem 6. The instance $\mathcal{P}$ of the PCP has a solution iff the ontology $\mathcal{O}_{\mathcal{P}}^{\prime}$ is not witnessed consistent.

Proof. Assume first that $\mathcal{P}$ has a solution $\mu=i_{1} \cdots i_{k}$ and let $u=v_{\mu}=w_{\mu}$ and $\mu^{\prime}=i_{1} i_{2} \cdots i_{k-1} \in\{1, \ldots, m\}^{*}$. Suppose there is a witnessed model $\mathcal{I}$ of $\mathcal{O}_{\mathcal{P}}^{\prime}$. Since $\mathcal{O}_{\mathcal{P}} \subseteq \mathcal{O}_{\mathcal{P}}^{\prime}, \mathcal{I}$ must also be a model of $\mathcal{O}_{\mathcal{P}}$. From Lemma 5 it then follows that there are nodes $\delta, \delta^{\prime} \in \Delta^{\mathcal{I}}$ such that $A^{\mathcal{I}}(\delta)=A^{\mathcal{I}_{\mathcal{P}}}(\mu)=B^{\mathcal{I}_{\mathcal{P}}}(\mu)=B^{\mathcal{I}}(\delta)$, $H^{\mathcal{I}}(\delta)=H^{\mathcal{I}_{\mathcal{P}}}(\mu)=q / 2$, and $r_{i_{k}}^{\mathcal{I}}\left(\delta^{\prime}, \delta\right)=1$. Then we have $((A \rightarrow B) \sqcap(B \rightarrow$ $A))^{\mathcal{I}}(\delta)=1$, and hence

$$
(((A \rightarrow B) \sqcap(B \rightarrow A)) \rightarrow H)^{\mathcal{I}}(\delta)=1 \Rightarrow q / 2=q / 2 .
$$

This then means that $\left(\forall r_{i_{k}} \cdot(((A \rightarrow B) \sqcap(B \rightarrow A)) \rightarrow H)\right)^{\mathcal{I}}\left(\delta^{\prime}\right) \leq q / 2$, violating one of the axioms in $\mathcal{T}_{\mathcal{P}}^{\prime} \backslash \mathcal{T}_{\mathcal{P}}$. Hence, $\mathcal{I}$ is cannot be a model of $\mathcal{O}_{\mathcal{P}}^{\prime}$.

Conversely, assume that $\mathcal{O}_{\mathcal{P}}^{\prime}$ is not witnessed consistent. Then $\mathcal{I}_{\mathcal{P}}$ is not a model of $\mathcal{O}_{\mathcal{P}}^{\prime}$. Since it is a model of $\mathcal{O}_{\mathcal{P}}$, there must exist an $i, 1 \leq i \leq m$ such that $\mathcal{I}_{\mathcal{P}}$ violates the axiom $\left\langle\top \sqsubseteq \forall r_{i} .(((A \rightarrow B) \sqcap(B \rightarrow A)) \rightarrow H) \geq 1\right\rangle$. This means that there is some $\mu \in\{1, \ldots, m\}^{*}$ such that

$$
\left(\forall r_{i} \cdot(((A \rightarrow B) \sqcap(B \rightarrow A)) \rightarrow H)\right)^{\mathcal{I}_{\mathcal{P}}}(\mu)<1 .
$$

Since $r_{i}^{\mathcal{I}_{\mathcal{P}}}\left(\mu, \mu^{\prime}\right)=0$ for all $\mu^{\prime} \neq \mu i$ and $r_{i}^{\mathcal{I}_{\mathcal{P}}}(\mu, \mu i)=1$, this implies that

$$
(((A \rightarrow B) \sqcap(B \rightarrow A)) \rightarrow H)^{\mathcal{I}_{\mathcal{P}}}(\mu i)<1 ;
$$

i.e. $((A \rightarrow B) \sqcap(B \rightarrow A))^{\mathcal{I}_{\mathcal{P}}}(\mu i)>q / 2$. From the equivalence $(1)$ above, it follows that $A^{\mathcal{I}_{\mathcal{P}}}(\mu i)=B^{\mathcal{I}_{\mathcal{P}}}(\mu i)$, and hence $\mu i$ is a solution of $\mathcal{P}$.

Corollary 7. Witnessed consistency of $*-\mathcal{A L E}$ ontologies is undecidable if conjunction is interpreted using a $t$-norm that $q$-starts with $\Pi$ for a rational number $q \in(0,1]$.

Notice that in the proofs of Lemma 5 and Theorem 6 , the second condition of the definition of witnessed models was never used. Moreover, the witnessed interpretation $\mathcal{I}_{\mathcal{P}}$ is also weakly witnessed. We thus have the following corollary.

Corollary 8. Weakly witnessed consistency and quasi-witnessed consistency of $*-\mathcal{A L E}$ ontologies are undecidable if conjunction is interpreted using a t-norm that $q$-starts with $\Pi$ for a rational number $q \in(0,1]$.

\section{$5 \quad$ Undecidability w.r.t. Strongly Witnessed Models}

Unfortunately, the model $\mathcal{I}_{\mathcal{P}}$ constructed in the previous section is not a strongly witnessed model of $\mathcal{O}_{\mathcal{P}}$ since, for instance, $\inf _{\eta \in \Delta^{\mathcal{I}_{\mathcal{P}}}}\left(\top^{\mathcal{I}_{\mathcal{P}}}(\eta) \Rightarrow A^{\mathcal{I}_{\mathcal{P}}}(\eta)\right)=0$, but there is no $\delta \in \Delta^{\mathcal{I}_{\mathcal{P}}}$ with $A^{\mathcal{I}_{\mathcal{P}}}(\delta)=0$. Thus, the construction of $\mathcal{O}_{\mathcal{P}}^{\prime}$ does not yield an undecidability result for strongly witnessed consistency in $*-\mathcal{A L E}$.

This means that we need a different reduction to prove undecidability of strongly witnessed consistency. This reduction will follow a similar idea to the 
one from the previous section, in which models describe a search for a solution of the PCP $\mathcal{P}$. However, rather than building the whole search tree, models will describe only individual branches of this tree. The condition (wit3) will help ensure that at some point in this branch a solution is found. Conversely, the models constructed from solutions will be finite, and thus trivially strongly witnessed.

Before describing the reduction in detail, we recall a useful property of $t$ norms. Using a t-norm $\otimes$ and its associated residuum $\Rightarrow$, one can express the minimum and maximum operators as follows [15]:

$-\min (x, y)=x \otimes(x \Rightarrow y)$,

$-\max (x, y)=\min (((x \Rightarrow y) \Rightarrow y),((y \Rightarrow x) \Rightarrow x))$.

We can thus introduce w.l.o.g. the $*-\mathcal{A L E}$ concept constructor max with the obvious semantics. We will use this constructor to simulate the non-deterministic choices in the search tree as described next.

Given an instance $\mathcal{P}=\left(\left(v_{1}, w_{1}\right), \ldots,\left(v_{m}, w_{m}\right)\right)$ of the PCP, we define the ABox $\mathcal{A}_{\mathcal{P}}^{0}$ and the TBox $\mathcal{T}_{\mathcal{P}}^{0}$ as in the previous section, and for every $i, 1 \leq i \leq m$, we construct the TBox

$$
\mathcal{T}_{\mathcal{P}}^{s i}:=\left\{\left\langle C_{i} \sqsubseteq \exists r_{i} \cdot \top \geq 1\right\rangle,\left\langle V_{i} \sqcap A^{(s+1)^{\left|v_{i}\right|}} \stackrel{r_{i}}{\rightsquigarrow} A\right\rangle,\left\langle W_{i} \sqcap B^{(s+1)^{\left|w_{i}\right|}} \stackrel{r_{i}}{\rightsquigarrow} B\right\rangle\right\} .
$$

The only difference between the TBoxes $\mathcal{T}_{\mathcal{P}}^{i}$ and $\mathcal{T}^{s i}$ is in the first axiom. Intuitively, the concept names $C_{i}$ encode the choice of the branch in the tree to be expanded. Only if $C_{i}^{\mathcal{I}}(\delta)=1$, there will be an $r_{i}$ successor with degree 1 , and the $i$-th branch of the tree will be explored. For this intuition to work, we need to ensure that at least one of the $C_{i} \mathrm{~s}$ is interpreted as 1 in every node. On the other hand, we can stop expanding the tree once a solution has been found. Using this intuition, we define the ontology $\mathcal{O}_{\mathcal{P}}^{s}:=\left(\mathcal{A}_{\mathcal{P}}^{s}, \mathcal{T}_{\mathcal{P}}^{s}\right)$ where

$$
\begin{aligned}
\mathcal{A}_{\mathcal{P}}^{s}:= & \mathcal{A}_{\mathcal{P}}^{0} \cup\left\{a: \max \left(C_{1}, \ldots, C_{m}\right)=1\right\}, \\
\mathcal{T}_{\mathcal{P}}^{s}:= & \mathcal{T}_{\mathcal{P}}^{0} \cup \bigcup_{i=1}^{m} \mathcal{T}_{\mathcal{P}}^{s i} \cup\{\langle(A \sqcap B) \rightarrow \perp \sqsubseteq \perp \geq 1\rangle\} \cup \\
& \left\{\left\langle\top \sqsubseteq \forall r_{i} \cdot \max \left((A \rightarrow B) \sqcap(B \rightarrow A), C_{1}, \ldots, C_{m}\right) \geq 1\right\rangle \mid 1 \leq i \leq m\right\} .
\end{aligned}
$$

Theorem 9. The instance $\mathcal{P}$ of the $P C P$ has a solution iff the ontology $\mathcal{O}_{\mathcal{P}}^{s}$ is strongly witnessed consistent.

Proof. Let $\nu=i_{1} i_{2} \cdots i_{k}$ be a solution of $\mathcal{P}$ and let pre $(\nu)$ denote the set of all prefixes of $\nu$. We build the finite interpretation $\mathcal{I}_{\mathcal{P}}^{s}$ as follows:

$-\Delta^{\mathcal{I}_{\mathcal{P}}^{s}}:=\operatorname{pre}(\nu)$

$-a^{\mathcal{I}_{\mathcal{P}}^{s}}=\varepsilon$,

for all $\mu \in \Delta^{\mathcal{I}_{\mathcal{P}}^{s}}$,

- $A^{\mathcal{I}_{\mathcal{P}}^{s}}(\mu)=q \cdot 2^{-v_{\mu}}, B^{\mathcal{I}_{\mathcal{P}}^{s}}(\mu)=q \cdot 2^{-w_{\mu}}$,

and for all $j, 1 \leq j \leq m$ 
$-V_{j}^{\mathcal{I}_{\mathcal{P}}^{s}}(\mu)=q \cdot 2^{-v_{j}}, W_{j}^{\mathcal{I}_{\mathcal{P}}^{s}}(\mu)=q \cdot 2^{-w_{j}}$,

$-C_{j}^{\mathcal{I}_{\mathcal{P}}^{s}}(\mu)=1$ if $\mu j \in \operatorname{pre}(\nu)$ and $C_{j}^{\mathcal{I}_{\mathcal{P}}^{s}}(\mu)=0$ otherwise, and

$-r_{j}^{\mathcal{I}_{\mathcal{P}}^{s}}(\mu, \mu j)=1$ if $\mu j \in \operatorname{pre}(\nu)$ and $r_{j}^{\mathcal{I}_{\mathcal{P}}^{s}}\left(\mu, \mu^{\prime}\right)=0$ if $\mu^{\prime} \in \operatorname{pre}(\nu)$ and $\mu^{\prime} \neq \mu j$.

We show now that $\mathcal{I}_{\mathcal{P}}^{s}$ is a model of $\mathcal{O}_{\mathcal{P}}^{s}$. Since $\mathcal{I}_{\mathcal{P}}^{s}$ is finite, it follows immediately that it is also strongly witnessed. Clearly $\mathcal{I}_{\mathcal{P}}^{s}$ satisfies all axioms in $\mathcal{A}_{\mathcal{P}}^{0}$; additionally, we have that $C_{i_{1}}^{\mathcal{I}_{\mathcal{P}}^{s}}(\varepsilon)=1$ and thus, $\mathcal{I}_{\mathcal{P}}^{s}$ satisfies $\mathcal{A}_{\mathcal{P}}^{s}$. The axiom $\langle(A \sqcap B) \rightarrow \perp \sqsubseteq \perp \geq 1\rangle$ expresses that $(A \sqcap B)^{\mathcal{I}_{\mathcal{P}}^{s}}(\mu) \Rightarrow 0=0$, and hence $(A \sqcap B)^{\mathcal{I}_{\mathcal{P}}^{s}}(\mu)>0$ for all $\mu \in \operatorname{pre}(\nu)$, which clearly holds. We now show that the other axioms are also satisfied for every $\mu \in \operatorname{pre}(\nu)$.

Let $\mu \in \operatorname{pre}(\nu) \backslash\{\nu\}$. Then we know that there exists $i, 1 \leq i \leq m$, such that $C_{i}^{\mathcal{I}_{\mathcal{P}}^{s}}(\mu)=1$ and $r_{i}^{\mathcal{I}_{\mathcal{P}}^{s}}(\mu, \mu i)=1$; thus $\mu$ satisfies the axioms in $\mathcal{T}_{\mathcal{P}}^{s_{\mathcal{P}}^{i}}$. Moreover, $C_{j}^{\mathcal{I}_{\mathcal{P}}^{s}}(\mu)=0=r_{j}^{\mathcal{I}_{\mathcal{P}}^{s}}\left(\mu, \mu^{\prime}\right)$ for all $j \neq i$ and all $\mu^{\prime} \in \operatorname{pre}(\nu)$, which means that $\mu$ trivially satisfies all axioms in $\mathcal{T}^{s}{ }_{\mathcal{P}}^{j}$. If $\mu i=\nu$, then $((A \rightarrow B) \sqcap(B \rightarrow$ $A))^{\mathcal{I}_{\mathcal{P}}^{s}}(\mu i)=1$ since $\nu$ is a solution. Otherwise, there is a $j, 1 \leq j \leq m$ with $\mu i j \in \operatorname{pre}(\nu)$, and thus $C_{j}^{\mathcal{I}_{\mathcal{P}}^{s}}(\mu i)=1$. Thus, we have in both cases that $\mu$ also satisfies the last axioms in $\mathcal{T}_{\mathcal{P}}^{S}$.

Finally, if $\mu=\nu$, then $r_{i}^{\mathcal{I}_{\mathcal{P}}^{s}}\left(\mu, \mu^{\prime}\right)=0$ and $C_{i}(\mu)=0$, for all $\mu^{\prime} \in \operatorname{pre}(\nu)$ and all $i, 1 \leq i \leq m$, and thus the axioms are all trivially satisfied.

Conversely, let $\mathcal{I}$ be a strongly witnessed model of $\mathcal{O}_{\mathcal{P}}^{s}$. Then, there must be an element $\delta_{0} \in \Delta^{\mathcal{I}}$ with $a^{\mathcal{I}}=\delta_{0}$. Since $\mathcal{I}$ must satisfy all axioms in $\mathcal{A}_{\mathcal{P}}^{s}$, there is an $i_{1}, 1 \leq i_{1} \leq m$ such that $C_{i_{1}}^{\mathcal{I}}\left(\delta_{0}\right)=1$. Since $\delta_{0}$ must satisfy the axioms in $\mathcal{T}^{s_{\mathcal{P}}^{i_{1}}}$, there must exist a $\delta_{1} \in \Delta^{\mathcal{I}}$ with $r_{i_{1}}^{\mathcal{I}}\left(\delta_{0}, \delta_{1}\right)=1, A^{\mathcal{I}}\left(\delta_{1}\right)=q \cdot 2^{-v_{i_{1}}}$, and $B^{\mathcal{I}}\left(\delta_{1}\right)=q \cdot 2^{-w_{i_{1}}}$. If $A^{\mathcal{I}}\left(\delta_{1}\right)=B^{\mathcal{I}}\left(\delta_{1}\right)$, then $i_{1}$ is a solution of $\mathcal{P}$. Otherwise, from the last set of axioms in $\mathcal{T}_{\mathcal{P}}^{\mathcal{s}}$, there must exist an $i_{2}, 1 \leq i_{2} \leq m$ with $C_{i_{2}}^{\mathcal{I}}\left(\delta_{1}\right)=1$. We can then iterate this construction to generate a sequence $i_{3}, i_{4}, \ldots$ of indices and $\delta_{2}, \delta_{3}, \ldots \in \Delta^{\mathcal{I}}$ where $A^{\mathcal{I}}\left(\delta_{k}\right)=q \cdot 2^{-v_{i_{1}} \cdots v_{i_{k}}}$, and $B^{\mathcal{I}}\left(\delta_{k}\right)=q \cdot 2^{-w_{i_{1}} \cdots w_{i_{k}}}$.

If there is some $k$ such that $A^{\mathcal{I}}\left(\delta_{k}\right)=B^{\mathcal{I}}\left(\delta_{k}\right)$, then $i_{1} \cdots i_{k}$ is a solution of $\mathcal{P}$. Assume now that no such $k$ exists. We then have an infinite sequence of indices $i_{1}, i_{2}, \ldots$ and since, for every $i, 1 \leq i \leq m$, either $v_{i} \neq 0$ or $w_{i} \neq 0$, then at least one of the sequences $v_{i_{1}} \cdots v_{i_{k}}, w_{i_{1}} \cdots w_{i_{k}}$ increases as $k$ gets larger. Thus, for every natural number $n$ there is a $k$ such that either $v_{i_{1}} \cdots v_{i_{k}}>n$ or $w_{i_{1}} \cdots w_{i_{k}}>n$; consequently $(A \sqcap B)^{\mathcal{I}}\left(\delta_{k}\right)<q \cdot 2^{-n}$. This implies that

$$
\inf _{\eta \in \Delta^{\mathcal{I}}}\left(\top^{\mathcal{I}}(\eta) \Rightarrow(A \sqcap B)^{\mathcal{I}}(\eta)\right)=0,
$$

and since $\mathcal{I}$ is strongly witnessed, there must exist a $\gamma \in \Delta^{\mathcal{I}}$ with

$$
0=\top^{\mathcal{I}}(\gamma) \Rightarrow(A \sqcap B)^{\mathcal{I}}(\gamma)=(A \sqcap B)^{\mathcal{I}}(\gamma) .
$$

But from this it follows that $((A \sqcap B) \rightarrow \perp)^{\mathcal{I}}(\gamma) \Rightarrow 0=0$, contradicting the axiom $\langle(A \sqcap B) \rightarrow \perp \sqsubseteq \perp \geq 1\rangle$ of $\mathcal{T}_{\mathcal{P}}^{\mathcal{S}}$. Thus, $\mathcal{P}$ has a solution.

Notice that, if $\mathcal{P}$ has no solution, then $\mathcal{O}_{\mathcal{P}}^{s}$ still has witnessed models, but no strongly witnessed models. It is also relevant to point out that $\mathcal{O}_{\mathcal{P}}^{s}$ has a 
strongly witnessed model iff it has a finite model. In fact, the condition of strongly witnessed was only used for ensuring finiteness of the model, and hence, that a solution is indeed found.

Corollary 10. For $*-\mathcal{A L E}$ ontologies, strongly witnessed consistency and consistency w.r.t. finite models are undecidable if conjunction is interpreted using a t-norm that $q$-starts with $\Pi$ for a rational number $q \in(0,1]$.

\section{Conclusions}

We have shown that consistency of $*-\mathcal{A L E}$ ontologies w.r.t. several notions of models, ranging from finite models to weakly witnessed models, is undecidable if the t-norm used to interpret conjunction is a t-norm that $q$-starts with $\Pi$ for a rational number $q \in(0,1]$. Since, for every $q \in(0,1]$, there exist uncountably many t-norms that $q$-start with $\Pi$, our results yield an uncountable family of tnorms for which reasoning in $*-\mathcal{A} \mathcal{L} \mathcal{E}$ becomes undecidable. Whether consistency in general (i.e., without restricting the class of interpretations) is also undecidable under these t-norms is still an open problem. The same is true if a t-norm that does not $q$-start with $\Pi$ for a rational number $q \in(0,1]$ is used. For the case of fuzzy DLs where disjunction and involutive negation is used in place of the residuum, we have an undecidability results for the product t-norm, but only for the case of witnessed models and with an extension of the TBox formalism to allow for the use of $>$ in fuzzy GCIs [2].

Since the results in $[5,2]$ have shown that the tableau-based algorithms for fuzzy DLs with GCIs are actually incorrect, the only decidability results for fuzzy DLs with GCIs that are currently available are those that use a finite set of fuzzy membership degrees $[11,12,10]$, or consider a rather simple t-norm (e.g. the Gödel t-norm) over the interval $[0,1]$, where only finitely many membership degrees are relevant for reasoning [6]. In these cases, a black-box approach that calls a crisp DL reasoner can be used.

\section{References}

1. F. Baader, D. Calvanese, D. McGuinness, D. Nardi, and P. F. Patel-Schneider, editors. The Description Logic Handbook: Theory, Implementation, and Applications. Cambridge University Press, 2003.

2. F. Baader and R. Peñaloza. Are fuzzy description logics with general concept inclusion axioms decidable? In Proc. of Fuzz-IEEE 2011, pages 1735-1742. IEEE Press, 2011.

3. F. Baader and U. Sattler. An overview of tableau algorithms for description logics. Studia Logica, 69:5-40, 2001.

4. F. Bobillo, F. Bou, and U. Straccia. On the failure of the finite model property in some fuzzy description logics. CoRR, abs/1003.1588, 2010.

5. F. Bobillo, F. Bou, and U. Straccia. On the failure of the finite model property in some fuzzy description logics. Fuzzy Sets and Systems, 172(23):1-12, 2011. 
6. F. Bobillo, M. Delgado, J. Gómez-Romero, and U. Straccia. Fuzzy description logics under Gödel semantics. Int. J. of Approx. Reas., 50(3):494-514, 2009.

7. F. Bobillo and U. Straccia. A fuzzy description logic with product t-norm. In Proc. of Fuzz-IEEE 200\%, pages 1-6. IEEE, 2007.

8. F. Bobillo and U. Straccia. On qualified cardinality restrictions in fuzzy description logics under Łukasiewicz semantics. In Proc. of IPMU-08, pages 1008-1015, 2008.

9. F. Bobillo and U. Straccia. Fuzzy description logics with general t-norms and datatypes. Fuzzy Sets and Systems, 160(23):3382-3402, 2009.

10. F. Bobillo and U. Straccia. Reasoning with the finitely many-valued Łukasiewicz fuzzy description logic $\mathcal{S R O} \mathcal{I} \mathcal{Q}$. Information Sciences, 181:758-778, 2011.

11. S. Borgwardt and R. Peñaloza. Description logics over lattices with multi-valued ontologies. In Proc. of IJCAI'11, 2011. To appear.

12. S. Borgwardt and R. Peñaloza. Fuzzy ontologies over lattices with t-norms. In Proc. of DL 2011, volume 745 of CEUR-WS, Barcelona, Spain, 2011.

13. M. Cerami, F. Esteva, and F. Bou. Decidability of a description logic over infinitevalued product logic. In Proc. of KR 2010. AAAI Press, 2010.

14. A. García-Cerdaña, E. Armengol, and F. Esteva. Fuzzy description logics and t-norm based fuzzy logics. Int. J. of Approx. Reas., 51:632 - 655, July/2010 2010.

15. P. Hájek. Metamathematics of Fuzzy Logic (Trends in Logic). Springer, 2001.

16. P. Hájek. Making fuzzy description logic more general. Fuzzy Sets and Systems, 154(1):1-15, 2005.

17. I. Horrocks, P. F. Patel-Schneider, and F. van Harmelen. From SHIQ and RDF to OWL: The making of a web ontology language. Journal of Web Semantics, $1(1): 7-26,2003$.

18. T. Lukasiewicz and U. Straccia. Managing uncertainty and vagueness in description logics for the semantic web. Journal of Web Semantics, 6(4):291-308, 2008.

19. R. Molitor and C. B. Tresp. Extending description logics to vague knowledge in medicine. In P. Szczepaniak, P. Lisboa, and S. Tsumoto, editors, Fuzzy Systems in Medicine, volume 41 of Studies in Fuzziness and Soft Computing, pages 617-635. Springer Verlag, 2000.

20. P. S. Mostert and A. L. Shields. On the structure of semigroups on a compact manifold with boundary. Annals of Mathematics, 65:117-143, 1957.

21. E. Post. A variant of a recursively unsolvable problem. Bulletin of the American Mathematical Society, 52:264-268, 1946.

22. G. Stoilos, G. B. Stamou, V. Tzouvaras, J. Z. Pan, and I. Horrocks. The fuzzy description logic f-SHIN . In Proc. of URSW'05, pages 67-76, 2005.

23. U. Straccia. Reasoning within fuzzy description logics. JAIR, 14:137-166, 2001.

24. U. Straccia. Description logics with fuzzy concrete domains. In Proc. of UAI'05, pages 559-567. AUAI Press, 2005.

25. U. Straccia and F. Bobillo. Mixed integer programming, general concept inclusions and fuzzy description logics. In Proc. of EUSFLAT 200\%, pages 213-220. Universitas Ostraviensis, 2007.

26. C. Tresp and R. Molitor. A description logic for vague knowledge. In Proc. of ECAI'98, pages 361-365, Brighton, UK, 1998. J. Wiley and Sons. 